\title{
Comparison of Gait Analysis by the Way of Semi-structured Interviews
}

\author{
Masaru Ohgiri ${ }^{1}$, Katsuma Yamada ${ }^{1}$, Hisanori Yuminaga ${ }^{2}$, Noriyuki Kida ${ }^{1}$, \\ and Hiroyuki Hamada ${ }^{1}$ \\ ${ }^{1}$ Kyoto Institute of Technology, Graduate School of Science and Technology, \\ Kyoto, Japan \\ summersoniclove@yahoo.co.jp, haagen-kattsu@nike.eonet.ne.jp, \\ \{hhamada, kida\}@kit.ac.jp \\ ${ }^{2}$ Department of Physical Therapy, Kansai Vocational College of Medicine, \\ Osaka, Japan \\ yuminaga@kansai.ac.jp
}

\begin{abstract}
In this study, we showed a video with five samples of patients walking to three practicing Physical Therapists. We used a one-on-one semistructured interview to investigate how the Physical Therapists would predict problems and plan treatment programs based on what they observed in the video, and then we analyzed the results. We obtained qualitative data from interviews and charted them as quantitative data for analysis, which is a new approach for motion observation.
\end{abstract}

Keywords: Posture Prediction and Analysis, semi-structured interview, gait analysis, physical therapy.

\section{Introduction}

According to article 2 of the law governing physical and occupational therapists, physical therapy is defined as, "To carry out treatment or regular exercise for a person with physical disabilities to restore basic motion ability, and to apply physical approaches such as electrostimulation, massage and thermal treatment." As mentioned in the law, physical therapists (hereinafter referred as PT) are required to improve the basic motion ability of their patients. Analysis and observation of basic motion is very important in clinical practice to comprehend the status of a patient in a limited amount of time. According to Usuda 1), exercise and motion analysis in the clinical practice of physical therapy plays the central role in evaluation and treatment, which is conducted by the PT. In general, qualitative analysis of motion patterns is performed. Based on the observed phenomenon, the PT infers the cause of exercise and motion dysfunctions through top-down thinking. According to Fukui 2), physical therapy is recognized as an applied science. The best way to become a good PT is to show strong interest in restoration and support of a patient while conducting motion analysis and accruing empirical value. 
However, under the present circumstances, the ability and method of motion observation varies depending on the PT. The approach to treatments derived from motion observation also differs. Thus, it is said that the therapeutic effect also differs depending on the accuracy of motion observation. For this reason, physical therapy is hardly considered a therapeutic action with scientific elements when it comes to assigning medical fees. The lack of standardization of motion observation methods has led to confusion among physical therapy students in clinical practices. In order to solve these problems, we will review the process of standardizing motion observation in physical therapy. Practical evidence-based physical therapy (EBPT) enhances the scientific validity of motion observation in physical therapy and is useful in educating students who aspire to become PT.

Suzuki 3) argues that it is important to not just observe patients but to comprehend "the order in which (the joints) move", "correlation of articular movement" and "displacement of joint angles per each motion." It is obvious that keen observation of motion is important, but there are many gray areas that are difficult to convey to the PT or PT training school students with less clinical experience. However there are some PTs with superior observation ability. They are generally referred to as skilled PTs with effective therapeutic abilities, and they can deduce the problem immediately upon observing the motion of a patient in clinical practice. An inexperienced PT with less experience in clinical practice who has a difficult time deducing problems from observing the motion of patients can receive guidance from a skilled PT and then offer effective treatments for patients. Every PT goes through this at some point. While recognizing the presence of skilled PTs, Bonkohara 4) reported that a PT's years of experience had no effect on their ability to observe and comprehend joint angle status per each phase of a walking patient. MacGinly 5) et al also reported that there is no connection between the experience and basic observation ability of PT.

The purpose of this study is to improve motion observation techniques for PTs and to establish the scientific validity of motion observation. We accumulated objective data based on a PT's motion observation in clinical practice using the qualitative research method of interviews and surveys. Then, we compared and discussed the factors involved in motion observation capability.

\section{$2 \quad$ Method}

\section{$2.1 \quad$ Subjects}

Of the three PTs who participated in this study; two are working at general hospitals and one is a teacher at a PT vocational training school. They are all practicing PTs and have six to eleven years clinical experience (the average years of experience is 8.6 years). Property of Subjects is shown in Table 1. Since this study is intended to accumulate the objective data from the thinking process of general PTs when it comes to observing motion, we selected the above mentioned PTs for the study with reference to the study of Yamada 6), et al. We verbally explained the purpose of the study and obtained their informal consent to cooperate with our research. 
Table 1. Property of Subjects

\begin{tabular}{ccccc}
\hline PT ID & Place of Work & Age & Gender & Years of Experience \\
\hline 1 & General hospital & 27 & Female & 6 years \\
2 & General hospital & 33 & Male & 9 years \\
3 & PT vocational training school & 40 & Male & 11 years \\
\hline
\end{tabular}

\subsection{Models for Walking Video}

The people in the walking video are actual patients. At the time of recording, rehabilitation was conducted at our hospital. We asked five patients who could walk safely during the video shooting to cooperate in this experiment. The name and duration of the diseases afflicting the model patients are shown in Table 2. Since our hospital has only an orthopedic surgery section, we selected patients with many opportunities to be treated in a clinic, such as patients with osteoarthritis or postoperative patients that have had surgery on their cervical region or lower limbs. Upon fully explaining the purpose of the study to the patients and obtaining their informal consent for cooperation in our research, we shot the video of the patients walking while taking into consideration the safety and stamina of the patients. The video recording was conducted in the rehabilitation room of our hospital. We set up the digital video camera (Nikon) at fixed points and recorded each patient walking along a 4-meter path twice. The filming was done from the front, back and side of the patient.

Table 2. Models for Walking Video

\begin{tabular}{rcccc}
\hline ID & Name of Disease & Age & Gender & Duration of Disease \\
\hline 1 & spondylosis deformans & 52 & male & 2 months \\
2 & osteoarthritis & 75 & male & 14 years \\
3 & ossification of posterior longitudinal ligament & 66 & male & 1 year \\
4 & femoral diaphyseal fracture & 40 & male & 9 months \\
5 & knee joint intraarticular fracture & 64 & female & 3 months \\
\hline
\end{tabular}

\subsection{Collection of Data}

The three participating PTs observed the video of patients walking, and then were each interviewed by a PT who is the head author of this paper with eight years of clinical experience as a PT. Since this study was designed to research the thinking process of PTs from observing action to planning a treatment program, we did not disclose any information about the patients' diagnoses or their course of rehabilitation beforehand in order to conduct the research in a non-biased way. Each of the PTs was able to watch the videos twice from the front and side angles. To avoid having the order of the videos affect the thinking process of the PTs, the interviewer randomly determined the order in which the videos were played.

The interview survey of the PTs was conducted by using a semi-structured interview technique. We performed interviews for about 10-15 minutes per subject. The interview time per PT is shown in Table 3. The semi-structured interview is a method 
to roughly determine questions in advance and ask more detailed questions during the flow of a casual conversation. This method allows us to obtain unadulterated answers to questions and carry out our investigation in a short time. In order to bring out the thinking process of the PT as much as possible, we applied a semi-structured interview technique rather than a structured interview that asks prepared questions without changing their orders. In a semi-structured interview, we are allowed to add questions according to the flow of a conversation.

In the interview, we asked the PTs, "By observing this video, what action do you focus on and how do you approach treatment?" We had the PTs reply as specifically as possible and added more questions based on the flow of the conversations such as, "Is there any other point that concerned you?" The conversation during the interview was recorded with the consent of the subjects. When identifiable personal information was stated during recording, we eliminated that part. The head author transcribed the conversations related to the physiotherapeutic thinking process.

Table 3. Interview time

\begin{tabular}{ccccc}
\hline & & \multicolumn{3}{c}{ Time(min.sec) } \\
\hline ID & Name of Disease & PT1 & PT2 & PT3 \\
\hline 1 & spondylosis deformans & 14.13 & 10.38 & 11.33 \\
2 & osteoarthritis & 13.24 & 12.30 & 17.29 \\
3 & ossification of posterior longitudinal ligament & 14.13 & 11.20 & 14.02 \\
4 & femoral diaphyseal fracture & 13.47 & 12.30 & 7.59 \\
5 & knee joint intraarticular fracture & 10.31 & 8.50 & 12.13 \\
& Total & 66.80 & 55.48 & 63.16 \\
\hline
\end{tabular}

\subsection{Categorization}

We labeled the speech data obtained in the interviews. Labeling was conducted for basically 1.Region, 2.Walking phase, 3.Physical function. When multiple regions or body functions were mentioned, we corresponded appropriately and labeled these statements in higher categories. For instance, the speech data referring to "the alignment of initial stance also shows strong abduction-external rotation of hip joint" consist of hip joint (1.region), initial stance (2.walking phase) and alignment

Table 4. List of Analyzed Category from Speech Data : Region x Time

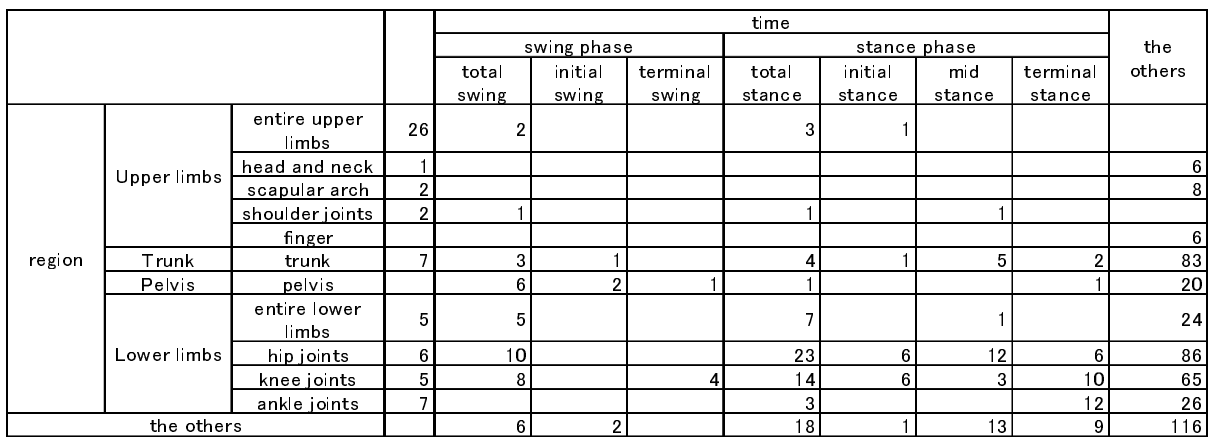


(3.physical function). It was defined as a single meaning unit (hip joint, initial stance, alignment). Another case that stated "the output of the gluteus medius is weak for the swinging left leg" was defined as a single meaning unit (hip joints, swing phase, muscle). We classified single meaning units according to similarity and divided them into groups. Then we categorized each group based on similarities among the groups. For categories formed through the extraction process mentioned above, we charted three patterns: 1.region x 2 walking phase, 1.region x 3. physical function, 2. walking phase $x$ 3. physical function. Then, we calculated the number of meaning units in each group (Table 4, 5, 6).

Table 5. List of Analyzed Category from Speech Data : Function x Region

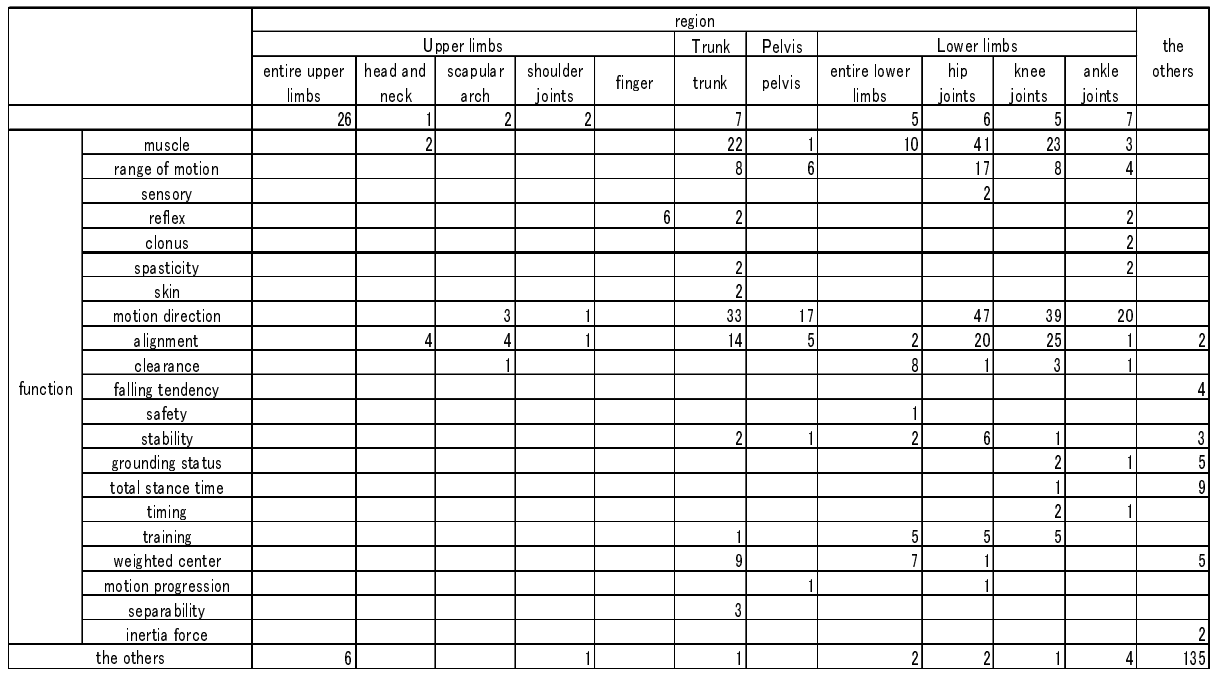

Table 6. List of Analyzed Category from Speech Data : Function x Time

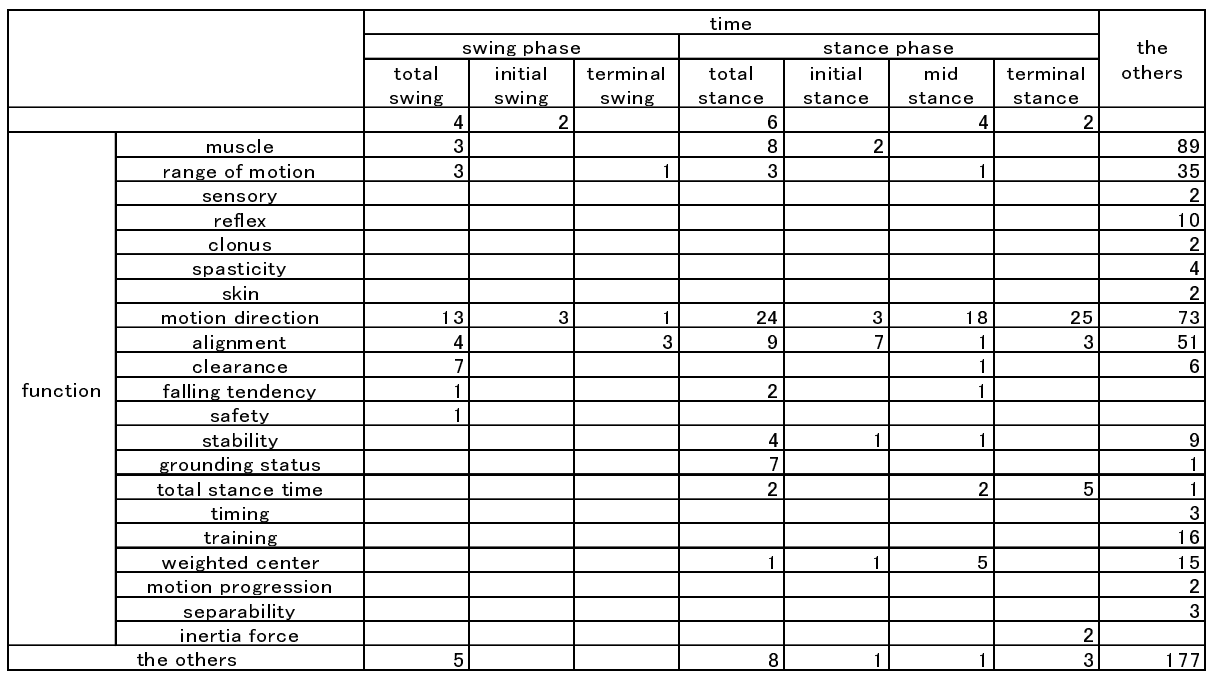




\section{$3 \quad$ Results}

First, we focused on the track of the marker attached to the handle of the pounding brush. The total speech time of the three subject PTs was 185 minutes (15 cases $\mathrm{x}$ several min. per each case), and the average speech time per person was 61.7 minutes (12.3 min. per each case). From speech content related to the physiotherapeutic thinking process, we extracted 716 meaning units for analysis. And we eventually categorized three main categories and eight sub-categories. Hereinafter, in this document, we indicate speech content with \lceil\rfloor , meaning unit with【】, sub-category with $\langle>$, main category with $\langle\gg$, and a lower category than sub-category with [].

There were three main categories: «region》, 《time», 《<unction》. The «region》 category indicated the physical parts that the PTs mentioned related to motion observation. Furthermore, the main category of «region» was divided into four sub-categories: $\langle$ upper limbs $>,\langle$ trunk $>,\langle$ pelvis $\rangle,\langle$ lower limbs $>$. These four sub-categories were also divided into eleven lower-categories: [entire upper limbs], [head and neck], [scapular arch], [shoulder joints], [finger], [trunk], [pelvis], [entire lower limbs], [hip joints], [knee joints], [ankle joints]. The 《time» category was limited to the speech contents referring to the walking phase observed by the PTs. 《TTime» was divided into two main categories: < swing phase $>$ and $<$ stance phase $>$. These two categories were divided into seven categories: [total swing], [initial swing], [terminal swing], [total stance], [initial stance], [mid stance] and [terminal stance]. The speech content referring to physical functions was categorized as 《function》. The main category of 《<unction》 was divided into two sub-categories: $<$ anatomical physiological function $>$ and $<$ mobility function $>$. These two sub-categories were divided into 21 lower categories: [muscle], [range of motion], [sensory], [reflex], [clonus], [spasticity], [skin], [motion direction], [alignment], [clearance], [falling tendency], [safety], [stability], [grounding status], [total stance time], [timing], [training], [weighted center], [motion progression], [separability], [inertia force].

\subsection{Region}

There were 551 meanings units referring to $<<$ region $>>$ among the 716 total meaning units. In the subcategories, 354 meaning units arose for $<$ lower limbs $>$, the most of all subcategories. Next was 106 meaning units for $<$ trunk $>$. The least-mentioned subcategory was <pelvis > with 31 units. In the lower category level of <<region>>, 149 meaning units were extracted for [hip joints], which got the most mentions. Next was 115 units for [knee joint]. The least mentioned lower category was [shoulder joints] with 5 units.

As for the meaning units on the PT level, 164 meaning units arose for PT1, 138 for PT2, and 251 units for PT3 (Table 7). On the PT level, the meaning units referring to $<$ lower limbs $>$ got the most mentions. On an individual basis, PT2 made more mention of [trunk] and [knee joints] than [hip joint]. The results for PT1 and PT2 were different. 
Table 7. Total Number of Meaning Units for Region (Per PT)

\begin{tabular}{cccc}
\hline & PT1 & PT2 & PT3 \\
\hline Upper limbs & 14 & 20 & 26 \\
Trunk & 26 & 33 & 47 \\
Pelvis & 12 & 9 & 10 \\
Entire lower limbs & 14 & 9 & 19 \\
Hip joint & 56 & 20 & 73 \\
Knee joints & 35 & 32 & 48 \\
Ankle joints & 7 & 13 & 28 \\
\hline
\end{tabular}

\subsection{Time}

Of the 716 total meaning units, 215 referred to $<<$ time $>>$. In the sub-categories, there were 164 meaning units for $<$ stance phase $>$ and 51 units for $<$ swing phase $>$. In the lower category level of $<<$ time $>>$, there were 74 meaning units for [total stance], which got the most mentions. Next was 41 units for [total swing]. And the least mentioned categories were 5 units for [initial swing] and [terminal swing], respectively.

On the PT level, 81 meaning units arose for PT1, 40 units for PT2 and 94 units for PT3 (Table 8). The meaning units referring to [total stance] got the most mentions on the PT level as well. For individual analysis, 32 meaning units referred to <swing phase $>$ arose for PT3, which was more than the 8 units for PT1 and 11 units for PT2.

Table 8. Total Number of Meaning Units for Time (Per PT)

\begin{tabular}{cccc}
\hline & PT1 & PT2 & PT3 \\
\hline Total swing & 8 & 6 & 27 \\
Initial swing & 0 & 5 & 0 \\
Terminal swing & 0 & 0 & 5 \\
Total stance & 26 & 7 & 41 \\
Initial stance & 3 & 4 & 8 \\
Mid stance & 29 & 3 & 3 \\
Terminal stance & 15 & 15 & 10 \\
\hline
\end{tabular}

\subsection{Function}

Of the 716 total meaning units, 503 referred to $<<$ function $>>$. In the sub-categories, $<$ mobility function $>$ received the most mentions, 338, followed by 165 for <anatomical physiological function $>$. In the lower categories for $<<$ function $>>$, the meaning units for [motion detection] had the most mentions, at 160, Next was 102 meaning units for [muscle]. The least mentioned categories were [sense], [clonus] and [inertia force] with 2 meaning units.

On the PT level, 166 meaning units arose for PT1, 117 units for PT2 and 220 units for PT3 (Table 9). <Physical function> got the most mentions on PT level as 
well. On an individual basis, PT3 mentioned [muscle] more than [motion direction], which showed the different tendency when compared to PT1 and PT2.

Table 9. Total Number of Meaning Units for Function (Per PT)

\begin{tabular}{cccc}
\hline & PT1 & PT2 & PT3 \\
\hline Muscle & 17 & 18 & 67 \\
Motion range of joints & 15 & 14 & 14 \\
Reflex & 0 & 0 & 10 \\
Motion direction & 64 & 48 & 48 \\
Alignment & 23 & 15 & 40 \\
Clearance & 6 & 1 & 7 \\
Weighted center & 9 & 9 & 4 \\
\hline
\end{tabular}

\subsection{Correlation between Region and Time}

Of the 716 total meaning units. 166 referred to $<<$ region $>>$ and $<<$ time $>>$. The distribution of all meaning units is shown in table 10. In the sub-categories, the meaning units referring to $<$ lower limbs $>\mathrm{x}<$ stance phase $>$ counted for 103 , which got the most mentions. The meaning units referring to $<$ pelvis $>\mathrm{x}<$ stance phase $>$, or $<$ upper limbs $>\mathrm{x}<$ swing phase $>$ were the least mentioned, accounting for 2-3 mentions.

On the PT level, 63 meaning units arose for PT1, 30 units for PT2 and 73 units for PT3. The results were similar in that the meaning units referring to <lower limbs $>\mathrm{x}<$ stance phase $>$ were mentioned most on the PT level as well. Also the meaning units referring to $<$ pelvis $>\mathrm{x}<$ swing phase $>$, and $<$ upper limbs $>\mathrm{x}<$ stance phase $>$ were least mentioned. On an individual basis, 27 meaning units arose for <upper limbs $>\mathrm{x}<$ swing phase $>$ and $<$ lower limbs $>\mathrm{x}<$ swing phase $>$ for PT3, which was more than the 7 for PT1 and 9 for PT2.

Table 10. Total Number of Meaning Units Referring to Region and Time

\begin{tabular}{ccc}
\hline & Swing phase & Stance phase \\
\hline Upper limbs & 3 & 6 \\
Trunk & 4 & 12 \\
Pelvis & 9 & 2 \\
Lower limbs & 27 & 103 \\
\hline
\end{tabular}

\subsection{Correlation between Region and Function}

Of the 716 total meaning units, 473 referred to $<<$ region $>>$ and $<<$ function $>>$. Distribution of all meaning units is shown in table 11. In the sub-categories, the meaning units referring to $<$ lower limbs $>\mathrm{x}<$ mobility function $>$ got the most mentions with 208 . The meaning units referring to $<$ upper limbs $>x<$ anatomical physiological function $>$ 
and $<$ pelvis $>\mathrm{x}<$ anatomical physiological function $>$ got the fewest mentions with 8 and 7 , respectively.

On the PT level, 150 meaning units arose for PT1, 114 units for PT2 and 209 units for PT3. The meaning units referring to $<$ lower limbs $>x<$ mobility function $>$ got the most mentions on the PT level as well. Only a few meaning units referred to <upper limbs $>x<$ anatomical physiological function $>$ and $<$ pelvis $>x<$ anatomical physiological function $>$.

Table 11. Total Number of Meaning Units Referring to Region and Function

\begin{tabular}{ccc}
\hline & $\begin{array}{c}\text { Anatomical physiological } \\
\text { function }\end{array}$ & $\begin{array}{c}\text { Mobility } \\
\text { function }\end{array}$ \\
\hline Upper limbs & 8 & 14 \\
Trunk & 36 & 62 \\
Pelvis & 7 & 24 \\
Lower limbs & 114 & 208 \\
\hline
\end{tabular}

\subsection{Correlation between Time and Function}

Of the 716 total meaning units, 179 meaning units referred to $<<$ time $>>$ and $<<$ function $>>$. Distribution of all meaning units is shown in table 12. In the sub-categories, $<$ stance phase $>x<$ mobility function $>$ got the most mentions with 125 . The least mentioned subcategory, with 7 meaning units, was $<$ swing phase $>x<$ anatomical physiological function>.

On the PT level, 74 meaning units arose for PT1, 31 units for PT2 and 74 units for PT3. Similarly, high distribution was observed for $<$ stance phase $>x<$ mobility function $>$. On an individual basis, 23 meaning units arose for $<$ swing phase $>x<$ anatomical physiological function $>$ and $<$ swing phase $>\mathrm{x}<$ mobility function $>$ for PT3, which was more than the 8 units for PT1 and 9 units for PT2.

Table 12. Total Number of Meaning Units Referring to Time and Function

\begin{tabular}{ccc}
\hline & $\begin{array}{c}\text { Anatomical physiological } \\
\text { function }\end{array}$ & $\begin{array}{c}\text { Mobility } \\
\text { function }\end{array}$ \\
\hline Swing phase & 7 & 33 \\
Stance phase & 14 & 125 \\
\hline
\end{tabular}

\section{Discussion}

In this study, we showed a video with five samples of patients walking to three practicing PTs. We used a one-on-one semi-structured interview to investigate how the PTs would predict problems and plan treatment programs based on what they observed in the video, and then we analyzed the results. The categories that arose, the number of meaning units and their distribution are discussed below.

The interview lasted 55 to 66 minutes for all five cases. No significant time difference was observed in each PT, even considering that the interview investigator had 
adjusted time. However, PT1 subjected 226 of the 716 total meaning units to analysis, compared with, 182 for PT2 and 306 for PT3. Significant differences were observed from PT to PT. Moreover, in the category $<<$ region $>>$ and subcategory [hip joints], 149 meaning units, the most in all categories, arose. 56 meaning units arose for PT1, 20 units for PT2 and 73 units. That figure for PT3 was almost a majority. Significant differences were observed from PT to PT. We continued to consider the category of [hip joint], which got the most meaning units from PT3.

Looking at the correlation between region $\mathrm{x}$ time for [hip joints] per PT, the distribution for PT1 was 1 for [swing], 9 for [stance], 3 for [initial stance], 11 for [mid stance], and 2 for [terminal stance]. The distribution for PT2 was 2 for [stance], 1 for [initial stance] and 2 for [terminal stance]. Neither PT mentioned [swing] and [mid stance], which are in the lower category of [hip joints]. The distribution for PT3 was 9 for [swing], 12 for [stance], 2 for [initial stance], 1 for [mid stance], 2 for [terminal stance]. In comparison with the other two PTs, the meaning units extracted for [swing] were decidedly more. For [swing], PT3 had 9 meaning units, while PT1 had only 1 and PT2 had none. Some of the comments referring to [swing] included, "What I am concerned about is that the flexion of hip joint hardly seems troubled when swinging out the right leg." or "If a patient can smoothly swing out his left leg, he can sustain his left lower limb by left hip joint adduction". The PT carefully observed not only [stance], [initial stance], [terminal stance] of [hip joint], but also [swing]. This indicates that their scope of observation had widened.

Looking at the correlation of region $\mathrm{x}$ function for [hip joint] per PT, the distribution for PT1 showed 7 for [muscle], 9 for [range of motion], 25 for [motion direction], 6 for [alignment], 1 for [clearance], 5 for [training] and 1 for [motion progression]. For PT2, there were 6 for [muscle], 4 for [range of motion], 7 for [motion direction], 1 for [alignment] and 1 for [weighted center]. No meaning units referred to [clearance], [training], [motion progression] in the lower category of [hip joint]. PT3 mentioned 28 for [muscle], 4 for [range of motion], 2 for [sensory], 15 for [motion direction], 13 for [alignment] and 5 for [stability]. In comparison with the other two PTs, he had more meaning units concerning [muscle], [motion direction] and [alignment].

Seven lower categories -- [initial stance], [initial stance], [terminal stance], [muscle], [range of motion], [alignment], [motion direction] -- were mentioned by all three PTs. When observing the motion of the hip joint, these seven categories mentioned have high objectivity as observation viewpoints. Each PT carefully observed [stance], requiring more resistance ability in walking motion compared to [swing], the activity of [muscle] around the hip joint such as the gluteus maximus muscle and the gluteus medius, flexion and extension of the hip joint, and [range of motion] and [motion direction] including internal/external rotation, adduction and abduction.

In the lower categories, no commonalities were observed among the three PTs. The meaning unit referring to [training] arose for PT1. Comments included, "Conduct training to change the motion from flexion to extension of hip joint", and "Incorporate training for one-leg support such as bridge motion by one leg". For the treatment program, she selected [training] as a necessary method upon considering [muscle] or [motion direction] of the [hip joint]. Meaning units referring to [stability] and [sense] 
arose for PT3. Sample comment for [stability] were, "The stability of hip joint is low" and "Take an approach to stabilize hip joint" These statements indicated that they were observing joint stability based on their observation of [hip joint] muscles, [motion direction] and [alignment]. Sample comments related to [sensory] included, "Sensory of hip joint is low" and "It is important to be conscious of hip joint". These statements indicated that they were conducting motion observation for [sensory] upon considering the [muscle] of [hip joint], [range of motion], [motion direction] and [alignment].

\section{Conclusion}

Since the distribution of meaning units in these categories resulted from interviews of PTs about their thinking processes after observing patients walking, the appearances of the main categories such as $<<$ region $>>$ and $<<$ function $>>$ were predictable. Moreover, the patients who participated in this study have in total one case of hip joint disease (femoral diaphyseal fracture), 2 cases of knee-joint disease (osteoarthritis, knee-joint intraarticular fracture) and 2 cases of (ossification of posterior longitudinal ligament, spondylosis deformans), it was predictable that the meaning units referring to [hip joint], [knee joints] and [trunk] occupied the top ranks in the lower category of $<<$ region $>>$. However, evaluating the distribution of meaning units in this category using charts is a novel approach. The quantitative research for motion observation has been scattered to some extent, and no study was found to capture qualitative data as quantitative data when it comes to motion observation analysis. Therefore, we obtained qualitative data from interviews and charted them as quantitative data for analysis, which is a new approach for motion observation.

Commonality in the low categories per each PT was clarified through charts. The charts also revealed the low categories with less commonality such as [training] for PT1, and [stability], [sense], [swing] for PT3. In the study of Brunnekreef 7), et al, they showed video of 30 patients with orthopedic disease to 10 PTs with different years of experience and had them fill in the structured form for walking analysis. Then they analyzed the intra-class and interobserver reliability, reporting that it was affected to a certain extent according to the clinical experience of the observer. This study also suggested the thinking process during motion observation differed according to years of experience. By increasing the scientific validity of this research, feedback was facilitated to confirm or compare with others during motion observation. With increasing scientific validity and facilitated feedback, this chart is useful for training inexperienced PTs or vocational training school students in motion observation.

The limitations of this study include the number of subjects, 3, and their years of experience, from 6 to 11 . Also five people participated as walking models, so it is hard to draw out generalizations. We therefore, will increase the number of PT subjects and walking models in future studies, considering these steps necessary to increase scientific validity. Although the result of this study is insufficient, there was no study to chart qualitative data as quantitative data from motion observation for comparison and analysis. We believe the result of this study helps comprehend the thinking process of PTs during motion observation. 


\section{References}

1. Shigeru, U., Eiki, T.: Scientific Verification of Exercise and Motion Analysis in Clinical. Physical Therapy 31(8), 483-488 (2004)

2. Tsutomu, F.: Clinical Movement Analysis and Kinetic Chain. Physical Therapy 36(8), 472-474 (2009)

3. Toshiaki, S., Takashi, N.: Motion Observation / Motion Analysis. Journal of Kansai Physical Therapy 3, 33-39 (2003)

4. Shuzou, B., Sumiko, Y.: Reliability and Accuracy of Observational Gait Analysis. Physical Therapy 23(6), 742-752 (2008)

5. MaGinly, J.L., Glodie, P.A., et al.: Accuracy and reliability of observational gait analysis data: Judgments of push-off in gait after stroke. Phys.Ther. 83, 146-160 (2003)

6. Yoichi, Y., Hitoshi, M.: Training Issues Arising from Physical Therapists' Self-recognition: A Survey of Physical Therapists Working in Medical Institutions. Physical Therapy 27(4), 385-389 (2012)

7. Brunnekreef, J.J., van Uden, C.J., et al.: Reliability of video-taped observational gait analysis in patients with orthopedic impairment. BMC Musculoskeletal Disorders 6(17), 1-9 (2005) 